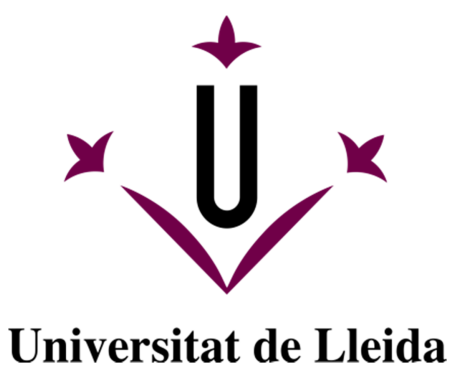

Document downloaded from:

http://hdl.handle.net/10459.1/63143

The final publication is available at:

https://doi.org/10.1080/14728222.2017.1369961

Copyright

(c) Taylor \& Francis, 2017 


\title{
New perspectives on CKD-induced dyslipidemia
}

\section{Marcelino Bermúdez-López ${ }^{1 *}$, David Arroyo ${ }^{1}$, Àngels Betriu ${ }^{1}$, Luis Masana ${ }^{2}$, Elvira Fernández $^{1}$, Jose $M$ Valdivielso ${ }^{1 *}$}

\author{
${ }^{1}$ Vascular and Renal Translational Research Group; Institute for Biomedical Research of Lleida \\ (IRBLleida); REDinREN del ISCIII, Lleida, Spain \\ 2 Unitat de Medicina Vascular i Metabolisme, Sant Joan University Hospital, IISPV, CIBERDEM, \\ Universitat Rovira I Virgili, Reus, Spain \\ * Corresponding authors:
}

Marcelino Bermúdez-López MD. PhD. E-mail: mbermudez@irblleida.cat José Manuel Valdivielso, PhD. E-mail: valdivielso@medicina.udl.es

Key Words: atherosclerosis, cardiovascular disease, chronic kidney disease, dyslipidemia, lipoproteins. 


\section{Abstract}

Introduction: chronic kidney disease (CKD) is a world-wide health concern associated with a significantly higher cardiovascular morbidity and mortality. One of the principal cardiovascular risk factors is the lipid profile. CKD patients have a more frequent and progressive atheromatous disease that cannot be explained by the classical lipid parameters used in the daily clinical practice.

Areas covered: the current review summarizes prevailing knowledge on the role of lipids in atheromathosis in CKD patients, including an overview of lipoprotein metabolism highlighting the CKD-induced alterations. Moreover, to obtain information beyond traditional lipid parameters, new state-of-the-art technologies such as lipoprotein subfraction profiling and lipidomics are also reviewed. Finally, we analyse the potential of new lipoprotein subclasses as therapeutic targets in CKD.

Expert opinion: the CKD-induced lipid profile has specific features distinct from the general population. Besides quantitative alterations, renal patients have a plethora of qualitative lipid alterations that cannot be detected by routine determinations and are responsible for the excess of cardiovascular risk. New parameters, such as lipoprotein particle number and size, together with new biomarkers obtained by lipidomics will personalize the management of these patients. Therefore, nephrologists need to be aware of new insights into lipoprotein metabolism to improve cardiovascular risk assessment. 


\section{Introduction}

Chronic kidney disease (CKD) is a complex clinical entity in which the function and structure of the kidneys are impaired. CKD is a global health issue with an increasing incidence and prevalence, mostly due to population aging and the rising frequency of diabetes, hypertension, and obesity. Progression of CKD towards end-stage renal disease (ESRD) represents a huge economic burden to healthcare systems since it increases the need for expensive renal replacement therapies [1, 2].

Over the past years, a large body of evidence strongly supports that CKD is accompanied by a significantly higher cardiovascular mortality, being cardiovascular disease (CVD) the primary cause of morbidity and premature mortality in renal patients. CKD patients show a higher incidence of coronary artery disease, myocardial infarction, congestive heart failure, cerebrovascular disease, peripheral arterial disease, and sudden cardiac death [3, 4]. In general terms, cardiovascular mortality rises as filtration rate declines. It is much higher in CKD stages 4-5 than in the general population, and markedly higher in ESKD as compared to earlier CKD stages [5].

Cardiovascular risk factors present in CKD can be classified as traditional or specific [6]. On the one hand, traditional risk factors that predict ischemic outcomes in the general population frequently coexist in renal patients. Interestingly, some of them do not show the same epidemiological behavior as in the general population, such as hypercholesterolemia, hypertension, and obesity [7]. On the other hand, specific risk factors that correlate with filtration rate reduction (such as anemia, inflammation and malnutrition) are also responsible for part of the cardiovascular risk excess in CKD patients [8]. One of the specific risk factors in which nephrologists have extensively focused their attention is vascular calcification, a phenomenon related to the very frequent alterations in mineral metabolism parameters found in CKD patients. These alterations, called chronic kidney disease-mineral and bone disorder (CKD-MBD), play a very important role in the physiopathology and progression of CVD in the CKD population. In fact, experimental evidence supports the existence of an important crosstalk between bone, kidney and the cardiovascular system [9].

Historically, vascular calcification had masked the relevance of atheromatosis in CKD, in part due to its high prevalence and the precocity of this complication. However, in the last decade atheromatosis and its clinical implications have gained importance in the renal population. The National Observatory of Atherosclerosis in Nephrology (NEFRONA) study, led by our group, was the first large longitudinal study describing the actual prevalence and associated risk factors of subclinical atheromatosis across 
different CKD stages [10, 11]. Patients with early CKD already have a higher prevalence of atheromatous plaques than those with normal renal function, and this prevalence is even higher in more advanced stages of CKD. Interestingly, a group of patients had plaques in femoral arteries but not in carotid arteries [12]. This highlights the importance of a wider ultrasound examination beyond carotid arteries to properly diagnose the disease. The results also show that the adjusted probability of having atheromatosis in CKD patients is $20-30 \%$ higher compared to matched controls with normal renal function [13]. Interestingly, CKD patients exhibit a more progressive and prevalent atheromatous disease, despite the fact that they lack the lipid profile commonly associated with atheromatosis in the general population [14]. Specific factors related to CKD have been identified as associated with atherosclerosis in this population [15-19]. However, the residual risk remains extremely high, and further research on risk factors affecting CKD-related atherosclerotic disease is urgently needed. Indeed a more precise characterization of the lipid profile seems a reasonable field for research.

Notwithstanding the growing scientific literature regarding cardiovascular risk factors, little progress has been made in the field of risk evaluation in patients with CKD. Given that lipid parameters currently measured in daily clinical practice do not explain the excess of atheromatous burden in renal patients, it is of paramount importance that the Nephrology community reconsiders the role of traditional lipid measurements to calculate cardiovascular risk in these patients. In contrast, nephrologists should consider the possibility of incorporating the use of new state-of-the-art technology that can provide information beyond traditional lipid parameters. In this review, we provide an overview of the normal lipoprotein metabolism, the lipid alterations induced by CKD, new approaches for lipoprotein characterization, and a brief description of the treatments of dislipemia in CKD, as well as our view on the importance of the new parameters of lipid metabolism with potential clinical research value in the renal population.

\section{A snapshot of lipoprotein metabolism}

Cholesterol esters and triglycerides are the two most important types of circulating lipids [20]. Due to their hydrophobicity, they need to be contained within particles called lipoproteins. They transport lipids from sites of absorption and/or synthesis to sites of consumption or processing. Based on their density, lipoproteins can be classified as chylomicrons, very-low-density lipoproteins (VLDL), intermediate-density lipoproteins (IDL), low-density lipoproteins (LDL), and high-density lipoproteins (HDL). They show 
significant differences in particle diameter, density, lipid components and apolipoprotein composition (figure 1). Lipoproteins have a hydrophobic core that contains cholesterol esters and triglycerides and a hydrophilic envelope formed by phospholipids, apolipoproteins and free unesterified cholesterol. Apolipoproteins have structural functions but also control lipoprotein metabolism through regulation of some enzymatic activities and receptor interactions (table 1).

Chylomicrons are triglyceride-rich lipoproteins (TGRLs) that are involved in absorption of exogenous dietary lipids. They are assembled by enterocytes, secreted into intestinal lymph and transported to bloodstream. Once in blood circulation, they are metabolized within minutes by the endothelium-associated lipoprotein lipase (LPL), thereby releasing free fatty acids (FFA), which are absorbed by the liver, muscle, and adipose tissues. As a consequence, a smaller, cholesterol-enriched particle, called chylomicron remnant is formed, which will be removed from circulation by the liver via the LDL receptor-related protein (LRP) [21].

VLDLs are TGRLs that transport endogenous triglycerides from the liver to peripheral tissues. LPL reduces their triglyceride content releasing FFA, which are absorbed by the liver, muscle, and adipose tissues. Therefore, VLDLs become smaller and relatively enriched in cholesterol esters forming IDLs. Then, IDLs are either removed from the bloodstream by the liver via LDLR or transformed into LDL due to a further LPL and Hepatic lipase $(\mathrm{HL})$ mediated hydrolysis. During these consecutive transformations, they accumulate cholesterol esters and their triglyceride content declines [22].

LDLs transport the major proportion of cholesterol to the liver and peripheral tissues. They originate from IDLs that have further reduced their triglyceride content by the LPL and $\mathrm{HL}$ (as previously described); or from HDL particles that have gained additional cholesterol esters in a transfer process mediated by the cholesterol ester transfer protein (CETP). The interaction of LDL particles with LDLR promotes their internalization, thereby removing them from the bloodstream. After endocytosis, the LDL particle is degraded, cholesterol cargo is released, and LDLR is recycled to the cell surface. The LDLR recycling can be compromised by the proprotein convertase subtilisin/Kexin type 9 (PCSK9), which fosters intracellular lysosomal degradation; hence, limiting LDLR abundance on the cell membrane and LDL internalizacion [23].

HDLs are the main players in reverse cholesterol transport from peripheral tissues to the liver. This process starts with the extraction of cholesterol from peripheral tissues in a process known as cholesterol efflux. The extracted free cholesterol is esterified by the lecithin-cholesterol acyltransferase (LCAT) protein and stored in the core of the 
HDL particle. This leads to the transformation of lipid-poor discoid HDL particles to spherical cholesterol-rich HDL particles, which then detach and are released into the bloodstream. In circulation, CETP transfers part of the cholesterol content of HDLs to IDLs and LDLs in exchange for their triglycerides. Finally, HDL particles are absorbed by the liver allowing lipid cargo release. After unloading of the lipid content, lipid-poor HDLs return to the circulation to repeat the cycle [2].

\section{A brief overview of CKD-induced dyslipidemia}

CKD patients show a dyslipidemia with distinct features from that of the general population. It is characterized by hypertriglyceridemia, varying levels of LDL cholesterol (LDL-C), an accumulation of TGRLs and low HDL cholesterol (HDL-C) levels [24]. The lipid profile has specific features depending on the CKD stage, proteinuria level, and type of renal replacement therapy $[25,26]$ (table 2). CKD impairs lipid metabolism in such a manner that all lipoprotein classes are altered to some extent (figure 2). Besides, renal patients have a plethora of qualitative lipid alterations that cannot be detected by routine determinations and has a strong impact on the progression of the disease.

TGRLs, such as chylomicrons, VLDL, and their remnants, accumulate in CKD patients from the earliest stages of renal dysfunction and show the highest levels in nephrotic syndrome and in peritoneal dialysis. This accumulation is the consequence of both a high production rate and a low catabolic rate [27]. Therefore, the artery wall is exposed to high plasma levels of TGRLs, which promotes atherogenesis.

IDLs, which are highly atherogenic, also accumulate in CKD patients due to: (i) a downregulation of hepatic lipase expression which impairs conversion of IDL into LDL [28]; and (ii) a reduced expression of LDLR and LRP in the liver that compromise particle removal [29].

Non-HDL cholesterol and Remnant cholesterol. The non-HDL cholesterol is cholesterol carried by ApoB-containing lipoproteins. Because any particle with a diameter below $70 \mathrm{~nm}$ can penetrate the artery wall, in situations characterized by an increased number of TGRL-derived particles such as CKD, they should be taken into account.

Cholesterol carried by non-HDL and non-LDL particles, is referred to "remnant cholesterol". It includes chylomicrons and VLDL remnants and IDLs. This group of particles is important in hypertriglyceridemia states because they accumulate and penetrate into the artery wall where they deliver high amounts of cholesterol. In fact, in 
hypertriglyceridemia states such as CKD, these particles transport more cholesterol than LDL particles. This must be taken into account in postprandial states, insulin resistance situations and in general, in all patients with an atherogenic dyslipidemia profile as CKD subjects.

LDL-C levels depend on the stage of CKD. Whereas in early stages patients show high LDL-C levels, in ESRD and particularly in hemodialysis they show normal or reduced concentrations [30]. Usually, renal patients show a reduced LDL particle catabolism and a decreased production of LDLs, resulting in nearly normal or reduced amounts. However, nephrotic syndrome is commonly associated with elevated LDL-C due to an overproduction of these particles. Beyond the quantitative difference, there is a significant qualitative modification of LDL particles in CKD. They become more atherogenic due to abnormal post-translational modification of their components. The oxidative environment associated with uremia favors several levels of oxidation, glycosylation and carbamylation that boost atherosclerosis [31].

Lipoprotein(a), also known as $L p(a)$, is an LDL-like molecule that contains an apolipoprotein(a) molecule attached to an LDL particle. Although many aspects of its metabolism remain elusive and its specific function remains unveiled, $L p(a)$ has demonstrated a pro-atherogenic effect [32]. In CKD, Lp(a) levels rises as filtration rate declines [33]. It has been correlated with coronary heart disease and cardiovascular mortality in hemodialysis patients [34]. An in vivo turnover study demonstrated a diminished clearance of $L p(a)$ in hemodialysis patients without a significant difference in the production rate compared to control patients [35].

HDL-C levels are usually reduced in renal patients. CKD compromises HDL metabolism in several manners. First, HDL maturation is limited due to a reduction of its apolipoproteins and LCAT levels, especially in dialysis patients [36]. Second, HDL particles also undergo abnormal post-translational modifications [37]. These alterations impair cholesterol efflux [38], anti-oxidative and anti-apoptotic functions, vasorelaxation, and inhibition of adhesion molecules expression [39]. Therefore, these modifications explain the diminished cardiovascular protective effect of HDLs observed in the CKD population.

Qualitative changes are very common in CKD. In renal patients, the antioxidant defenses are lowered leading to an enhanced oxidative stress [40]. This phenomenon appears almost at the beginning of CKD and increases as the filtration rate declines. Lipids and proteins are especially vulnerable to oxidation and this alteration alters their biological function. There are several oxidative modifications described in CKD. On the 
one hand, proteins can be carbonyled. This modification creates advanced glycation end products (AGEs) and advanced lipoxidation end products (ALEs) that accumulate in CKD. AGEs and ALEs have a strong impact on atherosclerosis since they favor LDL oxidation and display several pro-inflammatory effects, respectively [41, 42]. On the other hand, proteins can be carbamyled. Protein carbamylation is associated with CVD, mortality in CKD, affects lipoproteins and promotes atherosclerotic complications. Finally, cholesterol, phospholipids, fatty acids and lipoproteins are prone to oxidation. These modified molecules are very reactive and have a negative impact on body homeostasis [30].

\section{New insights into lipoprotein characterization}

As previously stated, CKD is associated with systemic inflammation, dyslipidemia, oxidative stress, accelerated atheromatosis and premature deaths from CVD [43]. Several aspects of this disease pointed towards the limited contribution of traditional lipid parameters. First, High LDL-C levels are associated with cardiovascular risk in the general population as well as in the CKD population. However, despite that most CKD patients show nearly normal or low LDL-C, data from the NEFRONA study indicate that the prevalence of atheromatosis was $68.7 \%$ and progressed in more than one half of patients with CKD after 24 months [13]. The multivariate logistic analysis revealed that, a part from triglyceride levels in CKD stage 3, there is not a clear association of traditional lipid parameters with the presence of atheromatous plaque [13]. Second, there is a considerable residual risk even at low levels of LDL-C in this population, particularly in ESRD patients. In fact, statins show a reduced benefit as CKD advances [44]. Altogether, these data indicate that the traditional lipid parameters are not at the forefront and therefore, a different approach is required. Modern detail analyses such as lipoprotein particle profiling, metabolomics and lipidomics may improve vascular risk assessment in this population.

\subsection{Particle number}

Besides LDL-C and HDL-C measurements that reflect the cholesterol mass within LDLs or HDLs, respectively, the actual lipoprotein particle number can be determined. In the past few years, although it is not a parameter that is determined in the daily clinical practice, particle number has demonstrated to be clinically relevant. Cardiovascular risk has been shown to be more closely related to LDL particle number (LDL-P) than LDL-C, especially when these parameters are discordant [45]. Indeed, two individuals having the same amount of LDL-C could have different cardiovascular risk if their LDL-P levels are different. Since the correlation of LDL-P with 
cardiovascular risk is more accurate than LDL-C, these two individuals have different probability to develop cardiovascular events, having more risk the one that has higher LDL-P levels. Multiple studies, such as VA-HIT, MESA, and JUPITER, also concluded that an elevated HDL particle number (HDL-P) predicted a reduced risk of coronary heart disease [46]. Therefore, particle number seems to be a stronger predictor of cardiovascular events than the classical routine lipid parameters.

Another approach to assess particle number is to quantify apolipoproteins. This is based on the fact that all potentially atherogenic lipoprotein particles (VLDL, IDL, LDL, and $L p(a)$ ) contain a single apolipoprotein $B(A p o B)$ molecule whereas anti-atherogenic HDL particles can contain several ApoA1 molecules. Therefore, it has been proposed that measuring apolipoproteins could improve cardiovascular risk assessment [47]. A recent study showed that ESKD patients show decreased levels of $A p o A 1$ and $A p o B$ compared to early stages [48].

\subsection{Lipoprotein size}

Nowadays, there are several techniques available to perform a deeper characterization that allows the separation of lipoproteins in different subfractions according to their size. Several studies have shown that small dense LDL particles are more proatherogenic than large LDL particles [49] because (i) small LDL particles have more ability to penetrate the endothelial cell barrier and accumulate beneath the intima; (ii) they are more easily oxidized; and, (iii) they remain in circulation for a longer time. It has been demonstrated an accumulation of small dense LDL particles in CKD, with a negative prognosis on cardiovascular events [50,51]. Regarding HDLs, high levels of large HDL particles correlate with less probability to develop atherosclerosis, while small HDL particles behave as a pro-atherogenic subclass [52]. Low levels of large HDL particles and high levels of small HDL particles correlate with a higher risk of incident cardiovascular events[53]. Curiously, two recent publications in CKD population showed unexpected results. First, the levels of small pro-atherogenic HDL particles decrease with CKD severity [48]. Second, haemodialysis patients have more of large HDL species, while small HDL species are more frequent in healthy people [54]. The clinical implications of such findings require further investigations.

Despite the extensively proven beneficial effect of statins reducing LDL-C levels and cardiovascular events, statins do not decrease the proportion of pro-atherogenic small dense LDL particles and do not reduce $L p(a)$ levels. Unfortunately, for long time, the only available therapeutic option to reduce both particles was plasmapheresis. Surprisingly, PCSK9 inhibitors, a part from having a potent effect on reducing LDL-C 
and cardiovascular events [55], they also decrease plasma Lp(a) levels [56] and have a strong impact on lipoprotein subfractions. They significantly reduce mean concentrations of total LDL-P, large and small LDL-P and total VLDL particles; and, unexpectedly, they increase HDL-P, being greater in large versus medium or small particles [57]. Therefore, although the clinical implications of these observations in the CKD population remain elusive, PCSK9 inhibitors could provide an extra benefit to these patients.

\subsection{Metabolomics \& lipidomics}

Unfortunately, none of the traditional lipid parameters is sensitive and specific enough by itself to evaluate cardiovascular risk in CKD. Based on that, the specific apolipoprotein and lipid composition are extremely valuable, since they can be used as novel biomarkers. New biomarker discovery requires advanced techniques such as metabolomics and lipidomics. Metabolomics is the systematic analysis of metabolites such as carbohydrates, amino acids, organic acids, nucleotides, lipids, and dipeptides in a given sample. Lipidomics, which is an essential part of metabolomics, aims to characterize the lipid species in a given sample [58].

Metabolomics has been used to evaluate potential biomarkers as well as therapeutic targets in many diseases such as cancer, metabolic diseases and neuropsychiatric diseases [59]. It provides information about the underlying cause, progression rate, and treatment response. Several excellent reviews reported metabolomic approaches applied to renal diseases $[60,61]$. Metabolomics is crucial to study uremic toxins, acute kidney injury, diabetic nephropathy, polycystic kidney disease, kidney cancer, and CKD [62]. Moreover, it is extremely valuable to evaluate nephrotoxicity of therapeutic drugs and environmental pollutants given its high sensitivity [63].

There are two main approaches. Non-targeted and targeted lipidomics. Non-targeted lipidomics aims to identify without bias as many lipids as possible. It is used in hypothesis-generating studies such as biomarker discovery [64]. On the contrary, targeted lipidomics aims to quantify a selected group of lipids exploring specific metabolic pathways or validating biomarkers previously identified using a non-targeted lipidomic profiling (discovery experiment) [65]. Since both approaches have pros and cons, most researchers choose to combine them. First, a non-targeted approach is conducted to identify as many candidates as possible in a discovery cohort. Then, targeted methods confirm previously identified compounds in a validation cohort to obtain more robust data [66]. Metabolomics and lipidomics data analyses require sophisticated statistical methods in order to show results in a meaningful way [67]. 
CKD has a strong effect on general metabolism. Over the last decade, hundreds of publications reported that CKD affects amino acids and their metabolites, nitric oxide, polyamines, urea cycle metabolites, uric acid, acylcarnitines, and lipids [68, 69]. The discovery of new biomarkers will generate a metabolomic signature that may improve early diagnosis of CKD, provide modern tools for monitoring progression of the disease and treatment response [70]. Metabolomics allows detection of dynamic changes in lipid metabolism before and after the onset of detectable changes in renal function or histology [71]. In that sense, the combination of some biomarkers, such as ricinoleic acid, stearic acid, cytosine, lysophosphatidic acid (LPA)(16:0), LPA(18:2), 3methylhistidine, and argininic acid allow the discrimination of patients with CKD from healthy subjects regardless of the underlying cause [72]. CKD patients accumulate total fatty acids, glycerolipids and glycerophospholipids that directly correlate with increased serum triglyceride levels and inversely correlate with estimated glomerular filtration rate (eGFR) [73]. In addition, detection of some of these lipid compounds such as monoacylglycerols and diacylglycerols can improve prediction of progression of CKD beyond eGFR and urine protein-creatinine ratio [74]. CKD also provokes the activation of inflammatory/oxidative pathways and suppression of antioxidant pathways that generate a specific metabolic fingerprint $[75,76]$.

Finally, in order to unveil currently unknown questions regarding CKD, an integrative system biology approach is required to combine all the information gathered from traditional lipid parameters, lipoprotein profiling and omic technologies [77].

\section{Therapeutic approach to CKD displipemia}

The management of dislipemia in renal patients has recently been reviewed [26, 78]. The Kidney Disease: Improving Global Outcomes (KDIGO) guidelines advise a therapeutic lifestyle change in adults with CKD. These changes include dietary modification, weight reduction, increased physical activity, reducing alcohol intake, and treatment of hyperglycemia if present. In addition, patients with high fasting TG serum levels $(>500 \mathrm{mg} / \mathrm{dL})$ should adopt a low-fat diet $(<15 \%$ total calories), reduce monosaccharide and disaccharide intake, as well as the total amount of dietary carbohydrates, and use fish oil to replace some long-chain TG [79].

It is recommended to evaluate lipid profile (TC, LDL-C, HDL-C, and TG) in adults with newly identified CKD. However, follow-up measurement of lipid levels is not required [79]. 
Some epidemiological studies revealed controversial results regarding lipid-lowering therapy and reduction of cardiovascular mortality in CKD patients. The SHARP study demonstrated that simvastatin and ezetimibe combination therapy was associated with lower risk of major atherosclerotic events compared to placebo in patients with CKD stage 3A-5 [80]. On the other hand, the 4D [81] and AURORA [82] trials conducted in patients treated with dialysis showed no positive effect on the primary composite endpoint of cardiovascular disease.

KDIGO guidelines recommend treatment with a statin or statin/ezetimibe combination in (i) adults aged $\geq 50$ years with eGFR $<60 \mathrm{~mL} / \mathrm{min} / 1.73 \mathrm{~m}^{2}$ who are not in dialysis or transplanted (GFR categories: G3a-G5); (ii) adults aged $\geq 50$ years with eGFR >60 $\mathrm{mL} / \mathrm{min} / 1.73 \mathrm{~m}^{2}$ (GFR categories: G1-G2); (iil) adults aged 18-49 years with CKD who are not in dialysis or transplanted, in the case of known coronary disease (myocardial infarction (MI) or coronary revascularization), diabetes, prior ischemic stroke or an estimated 10-year incidence of coronary death or non-fatal $\mathrm{MI}>10 \%$; and, (iv) adult kidney transplant recipients; On the contrary, in adults with dialysis-dependent CKD, statins or statin/ezetimibe combination is not recommended to be initiated. However, patients are recommended to continue their lipid-lowering therapy although dialysis treatment is initiated [79].

Finally, although hypertriglyceridemia is frequently present in CKD patients, fibric acid derivates are only used in cases of markedly elevated fasting TG (>1000 mg/dL) and should not be combined with statins because of potential toxicity [79].

\section{Conclusions}

The primary cause of morbidity and premature mortality in renal patients is cardiovascular disease. These patients show a higher prevalence of atheromatous disease from early stages, being even higher in advanced CKD stages. Surprisingly, although this population do not show the lipid profile commonly associated with atheromatosis in the general population, their atheromatous disease is more progressive.

The CKD-induced dyslipidemia shows hypertriglyceridemia, varying levels of LDL-C, an accumulation of TGRLs and low HDL-C. These parameters are modified depending on the stage of the disease, proteinuria levels, and the renal replacement therapy. CKD compromises lipid metabolism affecting all lipoprotein classes, creating a specific lipid profile in these patients. 
Besides the classical lipid alterations, recent approaches have revealed that there is a huge plethora of qualitative changes in lipoproteins. The combination of state-of-the-art techniques in metabolomics and lipidomics with advanced lipid tests such as lipoprotein subfraction profiling present an opportunity in order to progress towards precision medicine. These modern approaches will allow a better understanding of the CKD-induced dyslipidemia, they may also improve cardiovascular risk assessment, and it will eventually generate new therapeutic tools for cardiovascular disease prevention in patients with renal dysfunction. Therefore, translational research in this field must become a priority for nephrologists.

\section{Expert Opinion}

In the past few years, it has become evident that the information gathered from traditional lipid parameters shows important limitations in CKD patients because (i) they show a high prevalence of atheromatosis despite low LDL-C levels; (ii) there is not a clear association of other traditional lipid parameters with the presence of atheromatous plaque; and (iii) statins show a reduced benefit in advanced CKD stages, compared to early stages. Therefore, there is a clear need to find the real link between CKD-related lipid metabolism alterations and the excess of atheromatosis and cardiovascular disease in renal patients.

Several publications have highlighted the importance of a more detailed lipoprotein analysis. The importance of particle number and size has been extensively proven in the general population. In fact, the scientific community has accepted that lipoprotein subfraction profiling improves cardiovascular risk assessment compared to traditional lipid parameters. Unfortunately for nephrologists, there are very few studies of advanced lipoprotein profiling conducted in CKD patients and the clinical implication of such findings remain elusive. Therefore, clinical research in this field is urgently needed.

The potential of lipidology research in renal patients is unlimited since there are many questions to be answered. The ultimate goal in this field is to understand the impact of CKD on lipid metabolism and uncover the modifications responsible for the accelerated atheromatosis. Understanding the specific lipid alterations could enable the development of new therapeutic targets.

To achieve those goals clinicians need to be aware of new state-of-the-art techniques that will bring light to this field. One of the biggest challenges in the near future is the application of advanced lipoprotein test in the daily clinical practice. To our knowledge 
the future of lipidology will evolve together with the appearance of new techniques more accessible to clinicians that will allow a better characterization of lipid alterations.

\section{Article highlights}

1. CKD patients show a high cardiovascular mortality, being cardiovascular disease the primary cause of morbidity and premature mortality in renal patients.

2. CKD patients show a higher prevalence and a more progressive atheromatous disease that cannot be explained with the information gathered from the traditional lipid parameters.

3. The CKD-induced lipid profile is characterized by hypertriglyceridemia, varying levels of LDL cholesterol, and accumulation of TGRL and low HDL cholesterol levels.

4. Besides quantitative changes, there are many qualitative changes such as lipoprotein carbonylation, carbamylation and oxidation which are responsible from the excess of cardiovascular risk in this population.

5. New approaches such as lipoprotein profiling, metabolomics and lipidomics may improve cardiovascular risk assessment and personalize the treatment of these patients.

\section{Acknowledgments}

The authors want to apologize to all researchers whose work could not be cited due to limited extension of this review. The authors want to thank all members of the Vascular and Renal Translational Research Group of the Biomedical Research Institute of Lleida (IRBLleida) for the critical reading of the manuscript. This work was supported by the intramural program of the IRBLleida, the Instituto de Salud Carlos III (RETIC RD16/0009/0011, FIS PI16/01354) and FEDER funds. 


\section{Figure 1. Plasma lipoproteins.}

Major lipoproteins classes according to their density and size distribution (a). Their composition is shown in (b). Note that unlike HDL particles, chylomicrons are the biggest particles and show the lowest density. Abbreviations: HDL: high-density lipoprotein; IDL: intermediate-density lipoprotein; LDL: low-density lipoprotein; VLDL: very-low-density lipoprotein.

\section{Figure 2. Lipoprotein pathways and main modifications induced by CKD.}

CKD patients show an accumulation of VLDL, chylomicrons and their remnants due to a higher production and a lower catabolic rate. The reduced LPL activity reduces FFA release. IDLs also accumulate as a consequence of a downregulation of HL activity and a reduced expression of LDLR. The production and elimination of LDL particles are reduced and they suffer from abnormal modifications that increase their proatherogenic effect. HDL maturation is impaired in renal patients due to a reduced HDL precursor production, a reduced cholesterol efflux, and a reduced production and activity of LCAT. This leads to a reduced amount of spherical HDL particles and an increased amount of discoid HDL particles. Finally, the increased CETP levels reduce the amount of cholesterol present in HDL particles and increase their triglyceride content.

Abbreviations: CE: cholesterol ester; CETP: cholesterol ester transfer protein; CM: chylomicron; CMr: chylomicron remnant FFA: free fatty acids; HDL: high-density lipoprotein; HL: hepatic lipase; IDL: intermediate-density lipoprotein; LCAT: lecithin cholesterol acyltransferase; LDL: low-density lipoprotein; LDLR: LDL receptor; LPL: lipoprotein lipase; TG: triglyceride; VLDL: very-low-density lipoprotein. Figure adapted from Kwan et al. [2]. 
Table 1. Main types of plasma apolipoproteins

Apolipoprotein Structural Role

ApoA-I

Apo A-II

ApoA-IV

ApoA-V

ApoB-48

ApoB-100

Apo C-I

ApoC-II

ApoC-III

ApoD

ApoE

ApoM

Apo(a)

$\operatorname{Lp}(\mathrm{a})$ chylomicrons, HDL

chylomicrons, HDL

chylomicrons, HDL

chylomicrons, VLDL, HDL

Chylomicrons and remnant

VLDL, IDL, LDL, Lp(a)

chylomicrons, VLDL, IDL, HDL

chylomicrons, VLDL, IDL, HDL

chylomicrons, VLDL, IDL, HDL

Transport of small lipophilic molecules

chylomicrons, VLDL, IDL, HDL

LDLR ligand for chylomicron remnants; LRP ligand

Transport of small lipophilic molecules

Unknown

\section{Physiologic function}

LCAT activator

LPL regulator, LCAT and CETP cofactors

LCAT activator

LPL activator, chylomicron assembly

Structural component of chylomicrons

LDLR ligand

LCAT activator

LPL activator

LPL inhibitor, VLDL assembly
Unknown

Abbreviations: CETP: cholesterol ester transfer protein; HDL: high-density lipoprotein; IDL: intermediate-density lipoprotein; LCAT: lecithin cholesterol acyltransferase; LDL: low-density lipoprotein; LDLR: LDL receptor; Lp(a): lipoprotein(a); LPL: lipoprotein lipase; LRP: LDL receptor related protein; VLDL: very-low-density lipoprotein. 
Table 2. Traditional lipid parameter changes in CKD

\begin{tabular}{lcccc}
\hline Parameter & CKD 1-5 & Nephrotic syndrome & Haemodialysis & Peritoneal Dialysis \\
\hline Total cholesterol & $\nearrow$ & $\uparrow \uparrow$ & $\leftrightarrow \downarrow$ & $\uparrow$ \\
LDL cholesterol & $\nearrow$ & $\uparrow \uparrow$ & $\leftrightarrow \downarrow$ & $\uparrow$ \\
HDL cholesterol & $\downarrow$ & $\downarrow$ & $\downarrow$ & $\downarrow$ \\
Non-HDL cholesterol & $\nearrow$ & $\uparrow \uparrow$ & $\leftrightarrow \downarrow$ & $\uparrow$ \\
Triglycerides & $\nearrow$ & $\uparrow \uparrow$ & $\uparrow$ & $\uparrow$ \\
Lp(a) & $\nearrow$ & $\uparrow \uparrow$ & $\uparrow$ & $\uparrow \uparrow$
\end{tabular}

Plasma levels compared to healthy controls. Explanation of arrows: $\leftrightarrow:$ normal; $\uparrow:$ increased; $\uparrow \uparrow:$ markedly increased; $\lambda:$ increasing. Non-HDL cholesterol includes cholesterol in LDL, VLDL, IDL, chylomicrons and its remnants. Adapted from Kwan C.H et al. [2]. Abbreviations: HDL: highdensity lipoprotein; LDL: low-density lipoprotein; Lp(a): lipoprotein(a) 


\section{Bibliography}

1. Collister D, Ferguson T, Komenda P, et al. The Patterns, Risk Factors, and Prediction of Progression in Chronic Kidney Disease: A Narrative Review. Semin Nephrol 2016;36(4):273-282

2. Kwan BC, Kronenberg F, Beddhu S, et al. Lipoprotein metabolism and lipid management in chronic kidney disease. J Am Soc Nephrol 2007;18(4):1246-1261

3. Herzog CA, Asinger RW, Berger AK, et al. Cardiovascular disease in chronic kidney disease. A clinical update from Kidney Disease: Improving Global Outcomes (KDIGO). Kidney Int 2011;80(6):572-586

4. Whitman IR, Feldman HI, Deo R. CKD and sudden cardiac death: epidemiology, mechanisms, and therapeutic approaches. J Am Soc Nephrol 2012;23(12):1929-1939

5. Sarnak MJ, Levey AS, Schoolwerth AC, et al. Kidney disease as a risk factor for development of cardiovascular disease: a statement from the American Heart Association Councils on Kidney in Cardiovascular Disease, High Blood Pressure Research, Clinical Cardiology, and Epidemiology and Prevention. Circulation 2003;108(17):2154-2169 6. Balla S, Nusair MB, Alpert MA. Risk factors for atherosclerosis in patients with chronic kidney disease: recognition and management. Curr Opin Pharmacol 2013;13(2):192-199

7. Kalantar-Zadeh $\mathrm{K}$, Block $\mathrm{G}$, Humphreys $\mathrm{MH}$, et al. Reverse epidemiology of cardiovascular risk factors in maintenance dialysis patients. Kidney Int 2003;63(3):793-808

8. Filiopoulos V, Vlassopoulos D. Inflammatory syndrome in chronic kidney disease: pathogenesis and influence on outcomes. Inflamm Allergy Drug Targets 2009;8(5):369-382

9. Fujii H, Joki N. Mineral metabolism and cardiovascular disease in CKD. Clin Exp Nephrol 2017;21(Suppl 1):53-63

10. Junyent $\mathrm{M}$, Martínez $\mathrm{M}$, Borràs $\mathrm{M}$, et al. Predicting cardiovascular disease morbidity and mortality in chronic kidney disease in Spain. The rationale and design of NEFRONA: a prospective, multicenter, observational cohort study. BMC Nephrol 2010;11:14

11. Junyent $M$, Martínez $M$, Borrás $M$, et al. [Usefulness of imaging techniques and novel biomarkers in the prediction of cardiovascular risk in patients with chronic kidney disease in Spain: the NEFRONA project]. Nefrologia 2010;30(1):119-126

12. Arroyo D, Betriu A, Martinez-Alonso $M$, et al. Observational multicenter study to evaluate the prevalence and prognosis of subclinical atheromatosis in a Spanish chronic kidney disease cohort: baseline data from the NEFRONA study. BMC Nephrol 2014;15:168

13. Betriu A, Martinez-Alonso M, Arcidiacono MV, et al. Prevalence of subclinical atheromatosis and associated risk factors in chronic kidney disease: the NEFRONA study. Nephrol Dial Transplant 2014;29(7):1415-1422

14. Gracia M, Betriu À, Martínez-Alonso M, et al. Predictors of Subclinical Atheromatosis Progression over 2 Years in Patients with Different Stages of CKD. Clin J Am Soc Nephrol 2016;11(2):287-296

15. Martín M, Valls J, Betriu A, et al. Association of serum phosphorus with subclinical atherosclerosis in chronic kidney disease. Sex makes a difference. Atherosclerosis 2015;241(1):264-270

16. Fernández-Laso V, Sastre $C$, Valdivielso JM, et al. Soluble TWEAK levels predict the presence of carotid atherosclerotic plaques in subjects free from clinical cardiovascular diseases. Atherosclerosis 2015;239(2):358-363

17. Barrios C, Pascual J, Otero S, et al. Diabetic nephropathy is an independent factor associated to severe subclinical atheromatous disease. Atherosclerosis 2015;242(1):37-44 18. Fernández-Laso V, Méndez-Barbero N, Valdivielso JM, et al. Soluble TWEAK and atheromatosis progression in patients with chronic kidney disease. Atherosclerosis 2017;260:130-137

19. Anguiano L, Riera M, Pascual J, et al. Circulating angiotensin converting enzyme 2 activity as a biomarker of silent atherosclerosis in patients with chronic kidney disease.

Atherosclerosis 2016;253:135-143 
20. Nordestgaard BG, Varbo A. Triglycerides and cardiovascular disease. Lancet 2014;384(9943):626-635

21. Ginsberg HN. Lipoprotein physiology. Endocrinol Metab Clin North Am 1998;27(3):503-

519

22. Toth PP. Triglyceride-rich lipoproteins as a causal factor for cardiovascular disease. Vasc Health Risk Manag 2016;12:171-183

23. Glerup S, Schulz R, Laufs U, et al. Physiological and therapeutic regulation of PCSK9 activity in cardiovascular disease. Basic Res Cardiol 2017;112(3):32

24. Barter P. Lipoprotein metabolism and CKD: overview. Clin Exp Nephrol 2014;18(2):243-

246

25. Tsimihodimos V, Mitrogianni Z, Elisaf M. Dyslipidemia associated with chronic kidney disease. Open Cardiovasc Med J 2011;5:41-48

26. Hager MR, Narla AD, Tannock LR. Dyslipidemia in patients with chronic kidney disease. Rev Endocr Metab Disord 2016

27. Nestel PJ, Fidge NH, Tan MH. Increased lipoprotein-remnant formation in chronic renal failure. N Engl J Med 1982;307(6):329-333

28. Vaziri ND, Liang K. Down-regulation of tissue lipoprotein lipase expression in experimental chronic renal failure. Kidney Int 1996;50(6):1928-1935

29. Kim C, Vaziri ND. Down-regulation of hepatic LDL receptor-related protein (LRP) in chronic renal failure. Kidney Int 2005;67(3):1028-1032

30. Florens N, Calzada C, Lyasko E, et al. Modified Lipids and Lipoproteins in Chronic Kidney Disease: A New Class of Uremic Toxins. Toxins (Basel) 2016;8(12)

31. Samouilidou EC, Karpouza AP, Kostopoulos V, et al. Lipid abnormalities and oxidized LDL in chronic kidney disease patients on hemodialysis and peritoneal dialysis. Ren Fail 2012;34(2):160-164

32. Craig WY, Neveux LM, Palomaki GE, et al. Lipoprotein(a) as a risk factor for ischemic heart disease: metaanalysis of prospective studies. Clin Chem 1998;44(11):2301-2306

33. Kalra OP, Khaira A, Gambhir JK, et al. Lipoprotein (a) in chronic renal failure: effect of maintenance hemodialysis. Hemodial Int 2003;7(4):326-331

34. Kollerits B, Drechsler C, Krane V, et al. Lipoprotein(a) concentrations, apolipoprotein(a) isoforms and clinical endpoints in haemodialysis patients with type 2 diabetes mellitus: results from the 4D Study. Nephrol Dial Transplant 2016;31(11):1901-1908

35. Frischmann ME, Kronenberg F, Trenkwalder $\mathrm{E}$, et al. In vivo turnover study demonstrates diminished clearance of lipoprotein(a) in hemodialysis patients. Kidney Int 2007;71(10):1036-1043

36. Holzer M, Schilcher G, Curcic S, et al. Dialysis Modalities and HDL Composition and Function. J Am Soc Nephrol 2015;26(9):2267-2276

37. Shao B, Oda MN, Oram JF, et al. Myeloperoxidase: an inflammatory enzyme for generating dysfunctional high density lipoprotein. Curr Opin Cardiol 2006;21(4):322-328 38. Shah GM, Lin ZL, Kamanna VS, et al. Effect of serum subfractions from peritoneal dialysis patients on Hep-G2 cell apolipoprotein A-I and B metabolism. Kidney Int 1996;50(6):2079-2087

39. Shroff R, Speer T, Colin S, et al. HDL in children with CKD promotes endothelial dysfunction and an abnormal vascular phenotype. J Am Soc Nephrol 2014;25(11):2658-2668 40. Tucker PS, Dalbo VJ, Han T, et al. Clinical and research markers of oxidative stress in chronic kidney disease. Biomarkers 2013;18(2):103-115

41. Klein RL, Laimins M, Lopes-Virella MF. Isolation, characterization, and metabolism of the glycated and nonglycated subfractions of low-density lipoproteins isolated from type I diabetic patients and nondiabetic subjects. Diabetes 1995;44(9):1093-1098

42. Negre-Salvayre A, Coatrieux C, Ingueneau C, et al. Advanced lipid peroxidation end products in oxidative damage to proteins. Potential role in diseases and therapeutic prospects for the inhibitors. Br J Pharmacol 2008;153(1):6-20 
43. Navab KD, Elboudwarej O, Gharif $\mathrm{M}$, et al. Chronic inflammatory disorders and accelerated atherosclerosis: chronic kidney disease. Curr Pharm Des 2011;17(1):17-20 44. Barylski M, Nikfar S, Mikhailidis DP, et al. Statins decrease all-cause mortality only in CKD patients not requiring dialysis therapy--a meta-analysis of 11 randomized controlled trials involving 21,295 participants. Pharmacol Res 2013;72:35-44

45. Otvos JD, Mora S, Shalaurova I, et al. Clinical implications of discordance between lowdensity lipoprotein cholesterol and particle number. J Clin Lipidol 2011;5(2):105-113

46. Kontush A. HDL particle number and size as predictors of cardiovascular disease. Front Pharmacol 2015;6:218

47. Faergeman O. Introduction: Apolipoproteins and guidelines for prevention of cardiovascular disease. J Intern Med 2006;259(5):434-436

48. Honda $\mathrm{H}$, Hirano $\mathrm{T}$, Ueda $\mathrm{M}$, et al. High-Density Lipoprotein Subfractions and Their Oxidized Subfraction Particles in Patients with Chronic Kidney Disease. J Atheroscler Thromb 2016;23(1):81-94

49. Ip $\mathrm{S}$, Lichtenstein $\mathrm{AH}$, Chung $\mathrm{M}$, et al. Systematic review: association of low-density lipoprotein subfractions with cardiovascular outcomes. Ann Intern Med 2009;150(7):474-484

50. Chu M, Wang AY, Chan IH, et al. Serum small-dense LDL abnormalities in chronic renal disease patients. Br J Biomed Sci 2012;69(3):99-102

51. Shen H, Xu Y, Lu J, et al. Small dense low-density lipoprotein cholesterol was associated with future cardiovascular events in chronic kidney disease patients. BMC Nephrol 2016;17(1):143

52. Maeda $S$, Nakanishi S, Yoneda M, et al. Associations between small dense LDL, HDL subfractions (HDL2, HDL3) and risk of atherosclerosis in Japanese-Americans. J Atheroscler Thromb 2012;19(5):444-452

53. Asztalos BF, Collins D, Cupples LA, et al. Value of high-density lipoprotein (HDL) subpopulations in predicting recurrent cardiovascular events in the Veterans Affairs HDL Intervention Trial. Arterioscler Thromb Vasc Biol 2005;25(10):2185-2191

54. Gluba-Brzózka A, Franczyk B, Banach M, et al. Do HDL and LDL subfractions play a role in atherosclerosis in end-stage renal disease (ESRD) patients? Int Urol Nephrol 2017;49(1):155164

55. Sabatine MS, Giugliano RP, Keech AC, et al. Evolocumab and Clinical Outcomes in Patients with Cardiovascular Disease. N Engl J Med 2017;376(18):1713-1722

56. Reyes-Soffer G, Pavlyha M, Ngai C, et al. Effects of PCSK9 Inhibition With Alirocumab on Lipoprotein Metabolism in Healthy Humans. Circulation 2017;135(4):352-362

57. Koren MJ, Kereiakes D, Pourfarzib R, et al. Effect of PCSK9 Inhibition by Alirocumab on Lipoprotein Particle Concentrations Determined by Nuclear Magnetic Resonance Spectroscopy. J Am Heart Assoc 2015;4(11)

58. Murphy SA, Nicolaou A. Lipidomics applications in health, disease and nutrition research. Mol Nutr Food Res 2013;57(8):1336-1346

59. Zhao YY, Cheng XL, Vaziri ND, et al. UPLC-based metabonomic applications for discovering biomarkers of diseases in clinical chemistry. Clin Biochem 2014;47(15):16-26

60. Zhao YY. Metabolomics in chronic kidney disease. Clin Chim Acta 2013;422:59-69

61. Rhee EP. Metabolomics and renal disease. Curr Opin Nephrol Hypertens 2015;24(4):371-379

62. Weiss RH, Kim K. Metabolomics in the study of kidney diseases. Nat Rev Nephrol 2011;8(1):22-33

63. Zhao YY, Lint RC. Metabolomics in nephrotoxicity. Adv Clin Chem 2014;65:69-89 64. Vinayavekhin N, Saghatelian A. Untargeted metabolomics. Curr Protoc Mol Biol 2010;Chapter 30:Unit 30.31.31-24

65. Roberts LD, Souza AL, Gerszten RE, et al. Targeted metabolomics. Curr Protoc Mol Biol 2012; Chapter 30:Unit 30.32.31-24 
66. Wang $\mathrm{X}$, Wang $\mathrm{D}$, Wang $\mathrm{Y}$, et al. A combined non-targeted and targeted metabolomics approach to study the stereoselective metabolism of benalaxyl enantiomers in mouse hepatic microsomes. Environ Pollut 2016;212:358-365

67. Bartel J, Krumsiek J, Theis FJ. Statistical methods for the analysis of high-throughput metabolomics data. Comput Struct Biotechnol J 2013;4:e201301009

68. Hocher B, Adamski J. Metabolomics for clinical use and research in chronic kidney disease. Nat Rev Nephrol 2017

69. Breit M, Weinberger KM. Metabolic biomarkers for chronic kidney disease. Arch Biochem Biophys 2016;589:62-80

70. Chen $\mathrm{H}, \mathrm{Cao} \mathrm{G}$, Chen DQ, et al. Metabolomics insights into activated redox signaling and lipid metabolism dysfunction in chronic kidney disease progression. Redox Biol 2016;10:168-178

71. Zhao YY, Wang HL, Cheng XL, et al. Metabolomics analysis reveals the association between lipid abnormalities and oxidative stress, inflammation, fibrosis, and Nrf2 dysfunction in aristolochic acid-induced nephropathy. Sci Rep 2015;5:12936

72. Zhang ZH, Chen H, Vaziri ND, et al. Metabolomic Signatures of Chronic Kidney Disease of Diverse Etiologies in the Rats and Humans. J Proteome Res 2016;15(10):3802-3812

73. Chen H, Chen L, Liu D, et al. Combined Clinical Phenotype and Lipidomic Analysis Reveals the Impact of Chronic Kidney Disease on Lipid Metabolism. J Proteome Res 2017;16(4):1566-1578

74. Afshinnia F, Rajendiran TM, Karnovsky A, et al. Lipidomic Signature of Progression of Chronic Kidney Disease in the Chronic Renal Insufficiency Cohort. Kidney Int Rep 2016;1(4):256-268

75. Chen DQ, Cao $\mathrm{G}$, Chen $\mathrm{H}$, et al. Gene and protein expressions and metabolomics exhibit activated redox signaling and wnt/ $\beta$-catenin pathway are associated with metabolite dysfunction in patients with chronic kidney disease. Redox Biol 2017;12:505-521

76. Chen $\mathrm{DQ}$, Chen $\mathrm{H}$, Chen $\mathrm{L}$, et al. The link between phenotype and fatty acid metabolism in advanced chronic kidney disease. Nephrol Dial Transplant 2017

77. He JC, Chuang PY, Ma'ayan A, et al. Systems biology of kidney diseases. Kidney Int 2012;81(1):22-39

78. Mikolasevic I, Žutelija M, Mavrinac V, et al. Dyslipidemia in patients with chronic kidney disease: etiology and management. Int J Nephrol Renovasc Dis 2017;10:35-45

79. Wanner C, Tonelli M, Members KDIGOLGDWG. KDIGO Clinical Practice Guideline for Lipid Management in CKD: summary of recommendation statements and clinical approach to the patient. Kidney Int 2014;85(6):1303-1309

80. Baigent C, Landray MJ, Reith C, et al. The effects of lowering LDL cholesterol with simvastatin plus ezetimibe in patients with chronic kidney disease (Study of Heart and Renal Protection): a randomised placebo-controlled trial. Lancet 2011;377(9784):2181-2192

81. Wanner C, Krane V, März W, et al. Atorvastatin in patients with type 2 diabetes mellitus undergoing hemodialysis. N Engl J Med 2005;353(3):238-248

82. Fellström BC, Jardine AG, Schmieder RE, et al. Rosuvastatin and cardiovascular events in patients undergoing hemodialysis. N Engl J Med 2009;360(14):1395-1407

Papers of special note have been highlighted as either of interest (*) or of considerable interest $\left.{ }^{* *}\right)$ to readers.

30 ** A comprehensive review of lipid modifications and their implications in CKD.

32 ** A metaanalysis that highlights the role of lipoprotein (a) as a risk factor for ischemic heart disease. 
$44^{\star *}$ A metaanalysis that shows the effect of statins on all-cause of mortality only in CKd patients not in dialysis.

48** Publication that reveals HDL subfractions and their oxidized status in CKD patients

51** Publication that describes the implication of small dense LDL particles in cardiovascular events in CKD patients.

55** First clinical trial that demonstrates the effect of PCSK9 inhibitors in reducing LDL cholesterol and cardiovascular events. 
A

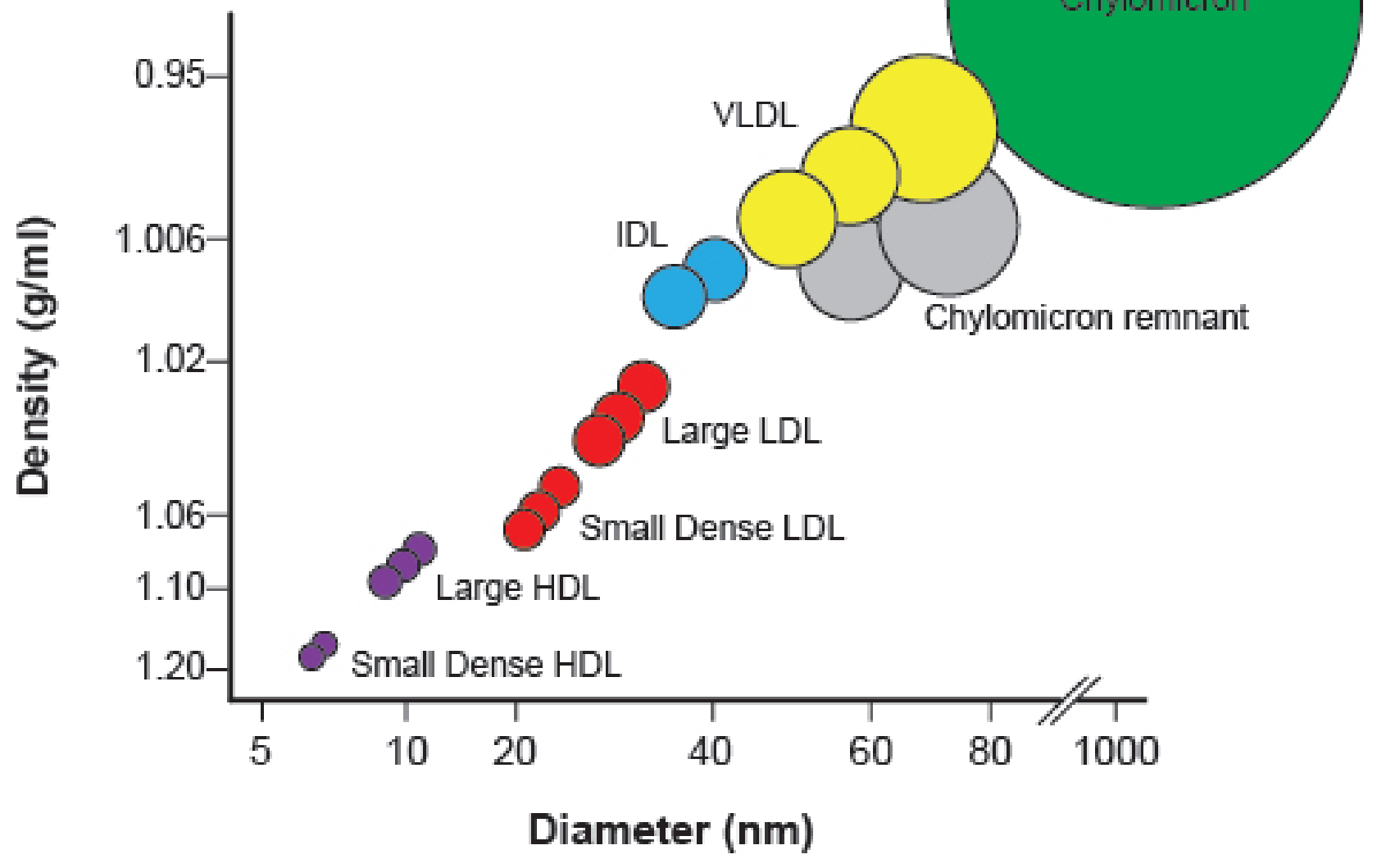

B

\section{Chylomicron}

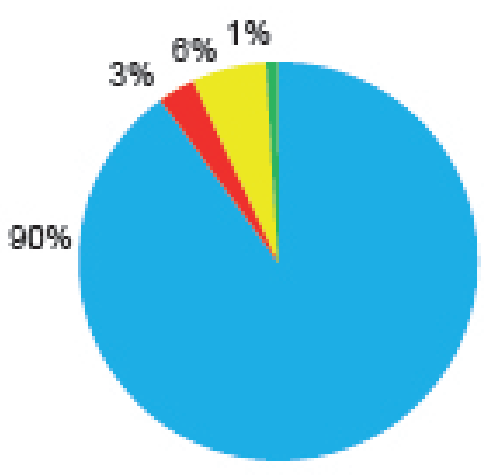

VLDL

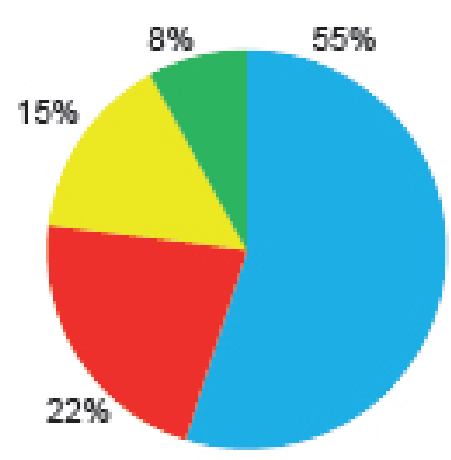

HDL

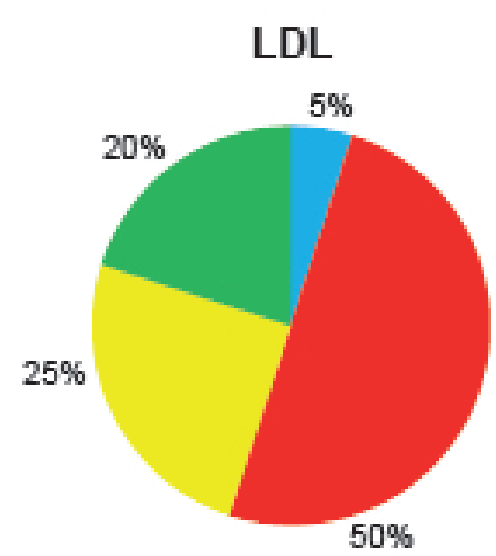

$5 \%$

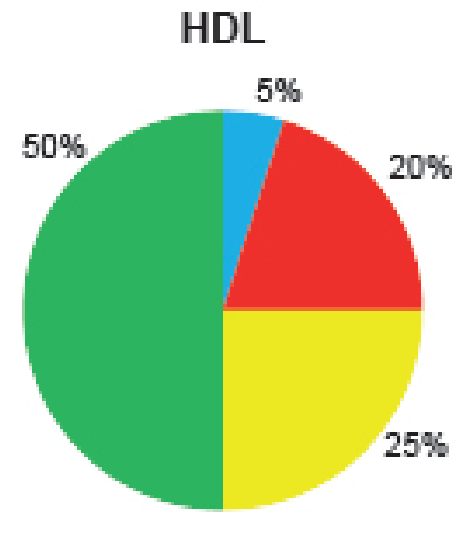

IDL

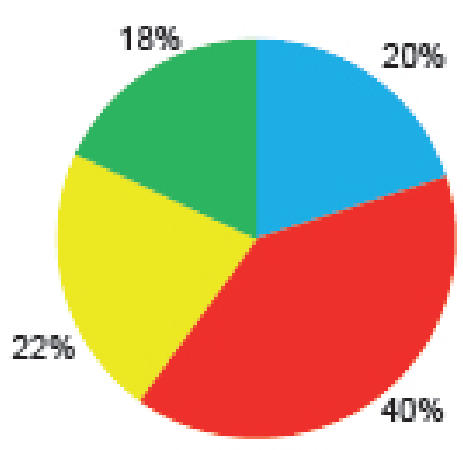

$40 \%$
Cholesterol

Triglycerides

Phospholipids

Proteins 
Figure 2

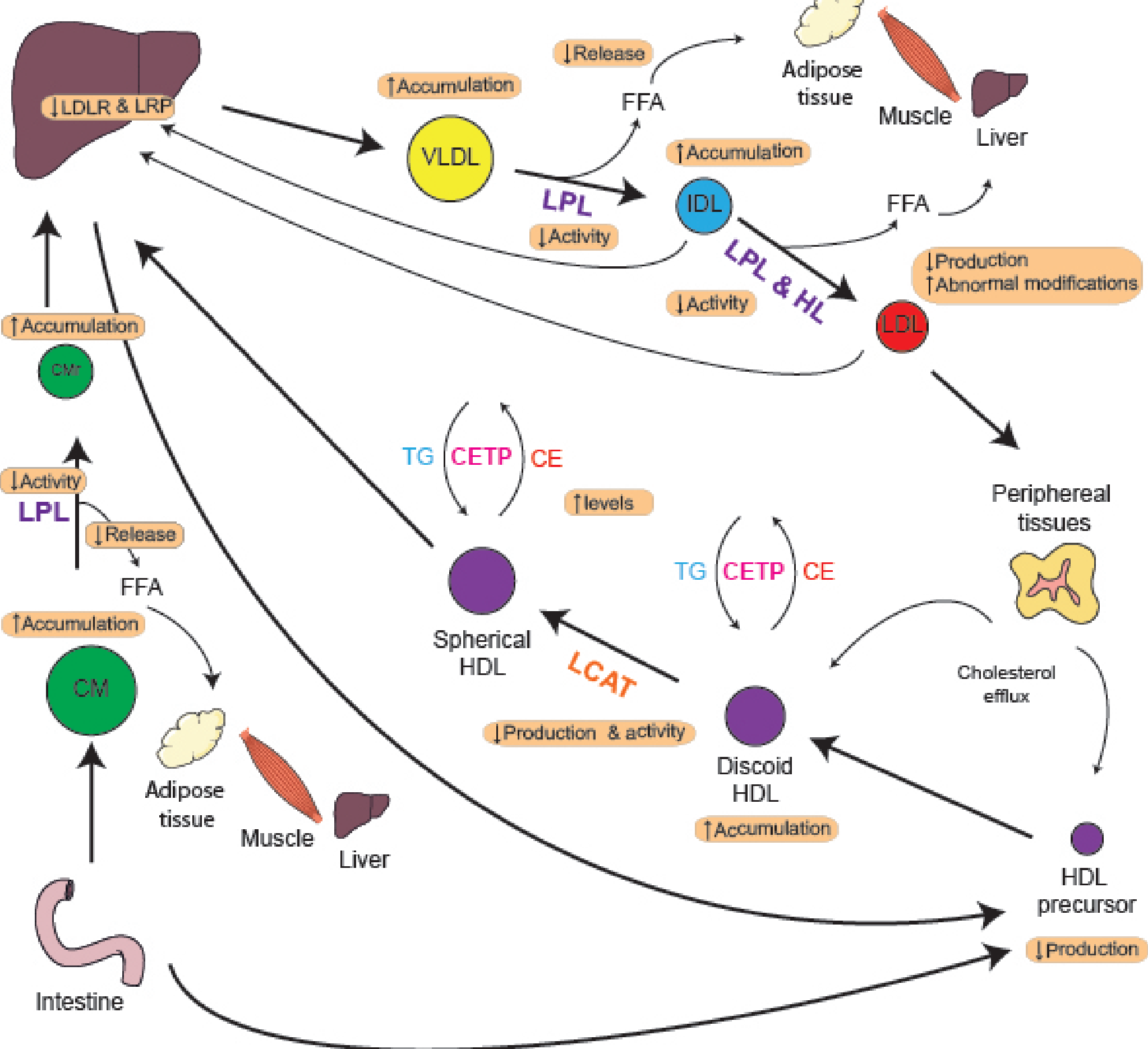

